\section{ORIGINAL RESEARCH}

\author{
A.M. Tokumaru \\ Y. Saito \\ S. Murayama \\ K. Kazutomi \\ Y. Sakiyama \\ M. Toyoda
}

M. Yamakawa

H. Terada

\title{
Imaging-Pathologic Correlation in Corticobasal Degeneration
}

BACKGROUND AND PURPOSE: The clinical diagnosis of corticobasal degeneration (CBD) is often difficult due to varied clinical manifestations. In 4 patients with neuropathologically confirmed CBD, characteristic imaging findings and correlations with neuropathologic features were evaluated. Furthermore, imaging findings in CBD were compared with neuropathologically confirmed progressive supranuclear palsy (PSP) for a differential diagnosis.

MATERIALS AND METHODS: Four patients with neuropathologically confirmed CBD were studied. We evaluated the area of the tegmentum in the midsagittal plane, subcortical white matter (SCWM) abnormality, asymmetric cerebral atrophy, and signal-intensity abnormality in the subthalamic nuclei on MR imaging and compared them with histopathologic findings. Then, MR imaging findings in CBD were compared with those in 13 patients with PSP.

RESULTS: On MR imaging, 3 patients had asymmetric cerebral atrophy extending to the central sulcus. On midsagittal sections, the mean midbrain tegmentum area was $66 \mathrm{~mm}^{2}$, being markedly smaller than normal, but there was no significant difference between PSP and CBD. All patients had signalintensity abnormalities of the SCWM, constituting primary degeneration neuropathologically; however, no diffuse signal-intensity abnormality in the SCWM existed in the 13 patients with PSP. In 3 patients, T1-weighted images showed symmetric high signal intensity in the subthalamic nuclei. Neuropathologically, these areas showed characteristic CBD. MR imaging signal-intensity changes also existed in 4 patients with PSP; however, subthalamic nucleus degeneration was more severe in PSP than in CBD.

CONCLUSIONS: In cases with midbrain tegmentum atrophy and signal-intensity changes in the subthalamic nuclei, the differential diagnosis distinguishing CBD from PSP based on MR imaging alone was difficult. White matter lesions and asymmetric atrophy can be useful for a differential diagnosis.

C orticobasal degeneration $(\mathrm{CBD})$ is a slowly progressive disorder with a clinically asymmetric onset characterized by apraxia, dystonia, postural instability, and an akinetic-rigid syndrome that does not respond to levodopa. However, clinical phenotypes of Alzheimer disease, Pick disease, and progressive supranuclear palsy (PSP) with similar characteristic features often make a differential diagnosis distinguishing these entities from CBD difficult in clinical practice.

Koyama et $\mathrm{al}^{1}$ recently reported asymmetric cerebral atrophy with dominance contralateral to the more clinically affected side, hyperintensity in the subcortical white matter (SCWM) in the frontotemporal area on fluid-attenuated inversion recovery (FLAIR), and atrophy of the midbrain tegmentum as new imaging findings of clinically diagnosed CBD, but no imaging findings in pathologically proved cases have been reported. ${ }^{3,4}$ We encountered 4 patients with neuropathologically confirmed CBD in whom the findings could be compared with those of MR imaging. Cortical symptoms were unclear in 3 , and it was difficult to make a diagnosis on the basis of clinical symptoms alone because of underlying dementia. Although the number of cases was small, because the

Received January 3, 2009; accepted after revision May 12.

From the Departments of Diagnostic Radiology (A.M.T., M.T., M.Y.), Neuropathology (S.M.), and Neurology (K.K.), Tokyo Metropolitan Medical Center of Gerontology, Itabashi-Ku, Tokyo, Japan; Department of Neuropathology (Y. Saito), National Center of Neurology and Psychiatry, Kodaira, Tokyo, Japan; Department of Neurology (Y. Sakiyama), Jichi Medical College, Omiya, Saitama, Japan; and Department of Radiology (H.T.), Toho University, Sakura Medical Center, Sakura, Chiba, Japan.

Please address correspondence to Aya M. Tokumaru, MD, PhD, Department of Radiology, Tokyo Metropolitan Medical Center of Gerontology, 35-2 Sakaecho, Itabashi-ku, Tokyo 173-0015, Japan; e-mail: tokumaru@tmghig.jp

DOI 10.3174/ajnr.A1721 neuropathology and images were collated in all cases, this study was significant with regard to the objectivity of the imaging findings associated with an accurate diagnosis. We also thought that it was important to identify differences in imaging findings between CBD and PSP, in which severe atrophy of the midbrain tegmentum has been reported. ${ }^{1,2}$ Therefore, MR imaging findings in $\mathrm{CBD}$ were compared with those in 13 cases of neuropathologically confirmed PSP.

\section{Materials and Methods}

\section{Patients}

Four patients (1 man, 3 women) with neuropathologically confirmed CBD were evaluated retrospectively. The patients' mean age at death was 70.8 years (range, 67-74 years). Table 1 lists the patient characteristics. The MR imaging findings in the patients with CBD (Table 2) were compared with those in 13 patients with neuropathologically confirmed PSP. All 13 patients with PSP were men, with a mean age at death of 78.3 years (range, $64-87$ years). For the comparative evaluation of atrophy of the midbrain tegmentum, 10 aged-matched control subjects (4 men and 6 women) with no neuropathologic degenerative disease or cerebrovascular disorder were selected (mean age at death, 74.6 years; range, $68-83$ years).

\section{MR Imaging Examinations}

All studies were performed with a 1.5T MR imaging unit (Signa Excite HD; GE Healthcare, Milwaukee, Wis). Axial T2-weighted images (TR/TE, 4300/89 ms; NEX, 2; FOV, 220 mm; section thickness, $5 \mathrm{~mm}$ with a section gap of $1 \mathrm{~mm}$ ) and FLAIR images (TR/TE, 10,002/106 ms; TI, $2500 \mathrm{~ms}$; NEX, 1; section thickness, $5 \mathrm{~mm}$ with a gap of $1 \mathrm{~mm}$ ), sagittal T1-weighted images (TR/TE, 600/14 ms; NEX, 2; section thickness, $5 \mathrm{~mm}$ with a gap of $1 \mathrm{~mm}$ ), or sagittal spoiled gradient-echo 


\begin{tabular}{|c|c|c|c|c|c|c|c|c|c|c|}
\hline $\begin{array}{l}\text { Case } \\
\text { No. }\end{array}$ & $\begin{array}{l}\text { Age at Onset } \\
\text { (yr) }\end{array}$ & Sex & $\begin{array}{c}\text { Duration } \\
\text { (yr) }\end{array}$ & Rigidity & Dystonia & $\begin{array}{l}\text { Pyramidal } \\
\text { Signs }\end{array}$ & $\begin{array}{c}\text { Cortical } \\
\text { Dysfunction }\end{array}$ & $\begin{array}{c}\text { Vertical } \\
\text { Gaze Palsy }\end{array}$ & Dementia & $\mathrm{CDx}$ \\
\hline 1 & 74 & $F$ & 10 & $\mathrm{Lt}>\mathrm{Rt}$ & - & - & Ocular apraxia & + & Mute & PSP? \\
\hline 2 & 68 & $\mathrm{~F}$ & 6 & $\mathrm{Lt}>\mathrm{Rt}$ & & & Apraxia (Lt hand) & \pm & + Severe akinetic mute & $A D$ \\
\hline 3 & 67 & $\mathrm{M}$ & 3 & $\mathrm{Rt}>\mathrm{Lt}$ & & & No cortical sign & + & + Severe & PDD \\
\hline 4 & 74 & $\mathrm{~F}$ & 6 & $\mathrm{Rt}>\mathrm{Lt}$ & - & - & - & + & + Severe & CBD \\
\hline
\end{tabular}

Note:-CBD indicates corticobasal degeneration; PSP?, progressive supranuclear palsy suspected; CDx, clinical diagnosis; AD, Alzheimer disease; PDD, Parkinson disease with dementia; - , no symptom; +, obvious symptom; \pm , suspicious symptom; Lt, left; Rt, right.

\begin{tabular}{|c|c|c|c|c|}
\hline \multirow[b]{2}{*}{$\begin{array}{l}\text { Case } \\
\text { No. }\end{array}$} & \multirow[b]{2}{*}{$\begin{array}{c}\text { Atrophy } \\
\text { (Dominant Cerebral Hemisphere) }\end{array}$} & \multicolumn{2}{|c|}{$\begin{array}{c}\text { White Matter Hyperintensity } \\
\text { on FLAIR }\end{array}$} & \multirow[b]{2}{*}{$\begin{array}{c}\text { Hyperintensity on T1WI in } \\
\text { Bil Subthalamic Nucleus }\end{array}$} \\
\hline & & $\begin{array}{l}\text { Precentral } \\
\text { Gyrus }\end{array}$ & $\begin{array}{l}\text { Frontal } \\
\text { Lobe }\end{array}$ & \\
\hline 1 & Rt frontal operculum and convexity & - & + & + \\
\hline 2 & Bil frontal convexity & - & + & + \\
\hline 3 & Lt frontoparietal & Bil & + & + \\
\hline 4 & Rt frontoparietal & Rt & + & + \\
\hline
\end{tabular}

Note:-FLAIR indicates fluid-attenuated inversion recovery; T1WI = T1-weighted imaging; Bil, bilateral; -, no signal abnormality; +, obvious signal abnormality.
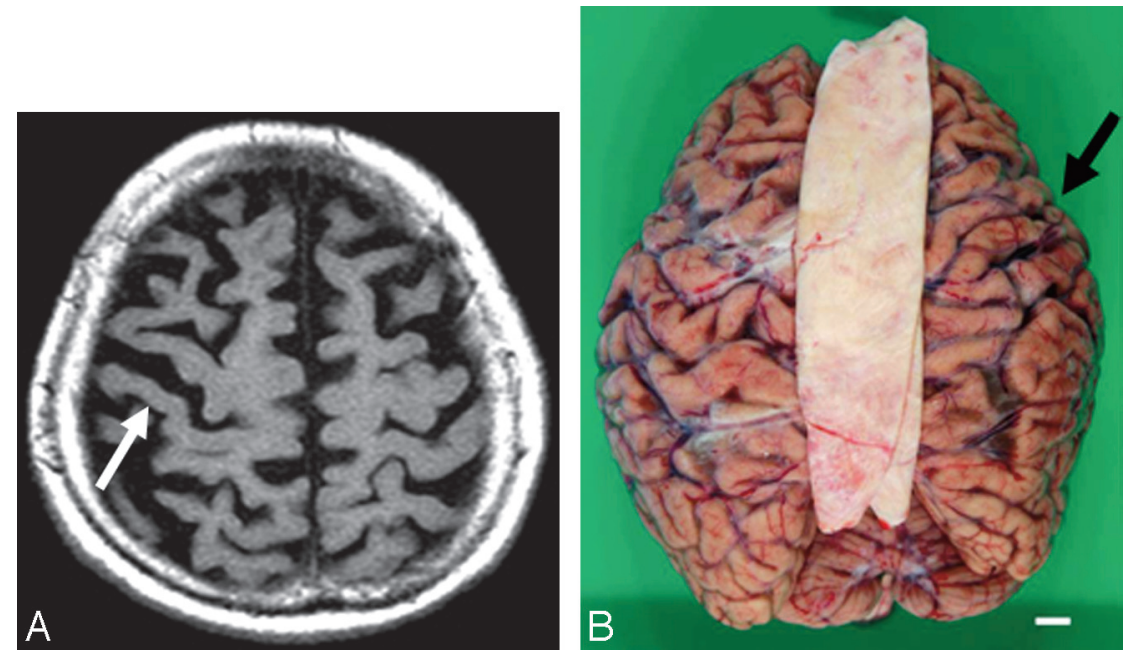

Fig 1. Corticobasal degeneration, case 1. An 84-year-old woman. A, Axial T2-weighted image shows right-side-dominant atrophy including the central sulcus (arrow). B, A macrospecimen of this patient shows right-frontal-dominant atrophy (arrow).

images (TR/TE, 21/6 ms; TI, $0 \mathrm{~ms}$; flip angle, 20 ${ }^{\circ}$ ) were obtained in all patients with CBD and PSP and healthy controls. Spin-echo coronal T1-weighted images (TR/TE, 600/14 ms; NEX, 2; section thickness, 5 $\mathrm{mm}$ with a gap of $1 \mathrm{~mm}$ ) were obtained in 3 of the 4 patients with $\mathrm{CBD}, 7$ of the 13 patients with PSP, and the 10 age-matched healthy controls. In 1 patient with CBD and 3 with PSP, T1-weighted coronal spin-echo images could not be obtained; spoiled gradient-echo imaging (TR/TE, 21/6 ms; TI, 0 ms; flip angle, $20^{\circ}$ ) was selected instead. These patients and 3 other patients with PSP without coronal sections were excluded from evaluation of the T1-weighted signal intensity. The area of the midbrain tegmentum was measured on an MR imaging workstation by using the method of Oba et $\mathrm{al}^{2}$ in a T1-weighted midsagittal section through the center of the interpeduncular cistern and the center of the cerebral aqueduct. Two neuroradiologists (A.M.T. and M.T.) performed these measurements blindly, twice at different times.

The localization and laterality of cerebral atrophy, signal intensity in the subthalamic nuclei, and signals in the SCWM were only qualitatively investigated because of the limitations of the retrospective nature of pathologically confirmed cases. The 2 neuroradiologists blindly investigated images of 4 patients with CBD, 13 patients with PSP, and 10 healthy controls twice at different times and visually evaluated the following points: 1) the presence or absence and laterality of cerebral atrophy and whether the atrophy included the central sulcus on T1-weighted imaging, 2) the presence or absence and localization of a high signal intensity in the SCWM on T2-weighted or FLAIR imaging, and 3) the presence or absence of an increase in the signal intensity in the subthalamic nuclei in the coronary view on T1-weighted imaging.

\section{Neuropathologic Examinations}

Informed consent for autopsy was obtained from all of the patients or their families. All serial autopsy cases were examined with the Brain Bank Aging Research protocol, irrespective of clinical diagnosis. ${ }^{3}$ At autopsy, after taking photographs of the whole brain, we serially sectioned the nondominant hemisphere or the hemisphere spared from focal lesions at a 7-mm thickness. The cerebrum was cut on the coronal plane; the brain stem, on the axial plane; and the cerebellum, on 

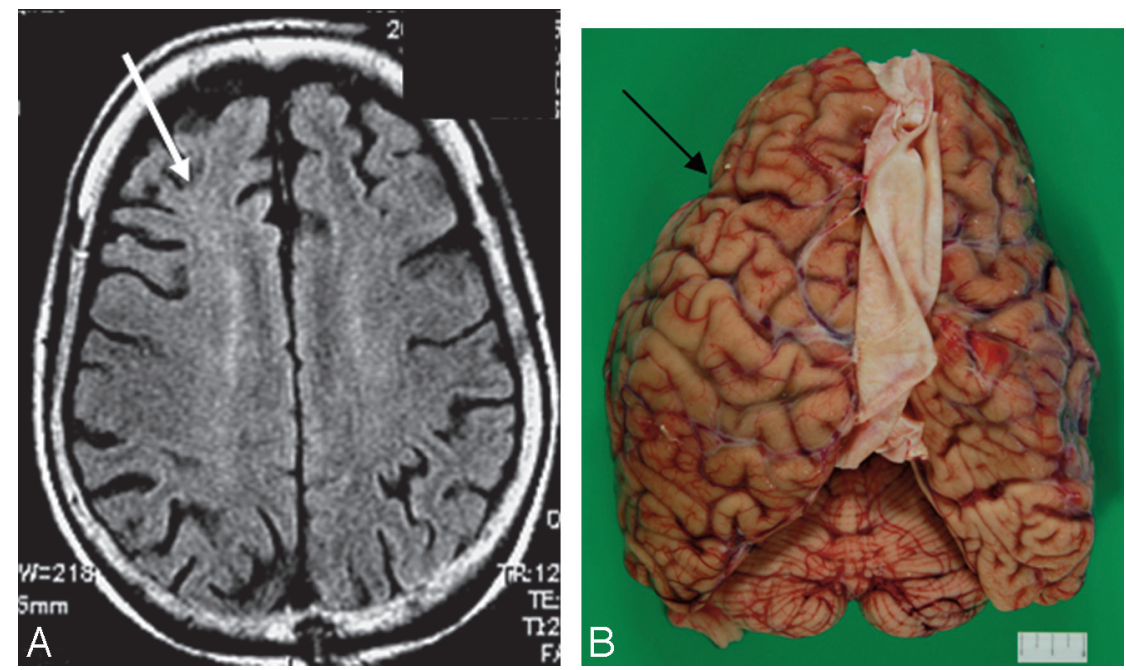

Fig 2. Corticobasal degeneration, case 2. A 74-year-old woman. A, An axial fluid-attenuated inversion recovery image 3 years before autopsy shows no obvious asymmetric atrophy Subcortical hyperintensity is shown in the right frontal white matter (white arrow). B, A macrospecimen of this patient shows mild frontal atrophy with some asymmetry (arrow).

the sagittal plane. Photographs were taken of all sections. Small pieces of the anterior amygdala; posterior hippocampus; frontal, temporal, and occipital poles; supramarginal gyrus; and rostral midbrain were directly fixed in $4 \%$ paraformaldehyde for 48 hours and prepared for immunohistochemical and ultrastructural studies. The remaining sections were quick frozen and stored at $-80^{\circ} \mathrm{C}$. The hemisphere kept for morphologic examinations was fixed in $20 \%$ neutral buffered formalin for 7-13 days and cut into 7-mm-thick sections, similar to those in the contralateral hemisphere. Paraffin-embedded sections of representative areas of the brain were examined.

The selected anatomic structures included those recommended by the Consortium to Establish a Registry for Alzheimer Disease, ${ }^{4}$ the Consensus Guidelines for the Diagnosis of Dementia with Lewy Bodies, ${ }^{5,6}$ Braak and Braak's recommendation, ${ }^{7}$ and the Diagnostic Criteria of Corticobasal Degeneration and Progressive Supranuclear Palsy. ${ }^{8,9}$ These included the frontal pole; temporal pole; cingulate gyrus; second frontal gyrus; accumbens and septal nuclei; amygdala; basal nucleus of Meynert; second temporal gyrus; anterior hippocampus with entorhinal and transentorhinal cortices; basal ganglia and hypothalamus with mamillary bodies; subthalamic nucleus; posterior hippocampus; thalamus with the red nucleus; motor cortex; parietal lobe with the intraparietal sulcus; visual cortex; midbrain; upper and middle pons; medulla oblongata; cerebellar vermis; dentate nucleus; and multiple cervical, thoracic, and lumbar levels of the spinal cord.

Six-micrometer-thick sections were routinely stained with hematoxylin-eosin and the Klüver-Barrera method. Selected sections were stained with the modified methenamine, Gallyas-Braak, and Bielschowsky silver staining for age-related changes, with Congo red for amyloid $\beta$ deposition and elastica-Masson trichrome staining for vascular changes.

\section{Immunohistochemistry}

Six-micrometer-thick serial sections were immunohistochemically stained by using a 20NX autostainer (Ventana, Tucson, Ariz), as previously described. The antibodies applied to all the cases were the following: antiphosphorylated $\alpha$-synuclein (psyn); phosphorylated tau (ptau) (AT8, monoclonal; Innogenetic, Temse, Belgium); 3-repeat tau (RD3, amino acids 209-224, monoclonal, Upstate; Millipore, Lake Placid, NY); 4-repeat tau (RD4, amino acid 275-291, monoclonal, Upstate; Millipore); amyloid $\beta$ 11-28 (12B2, monoclo-

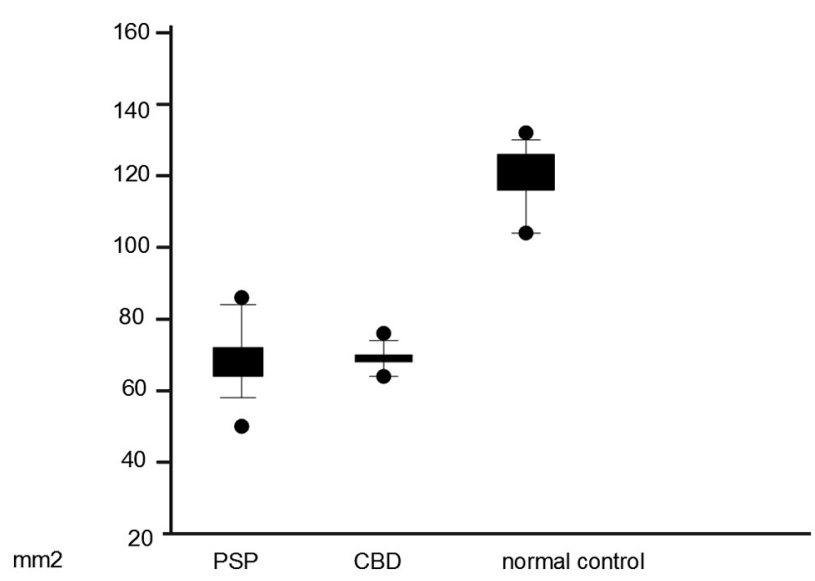

Fig 3. Scatterplot (mean, SD, and range) of the area of the midbrain in patients with progressive supranuclear palsy (PSP), corticobasal degeneration (CBD), and age-matched healthy controls. There was no individual overlap of the midbrain tegmental area between the healthy controls and patients with CBD and PSP, apparently showing that severe atrophy of the midbrain tegmentum was present in patients with CBD and PSP.

nal; IBL, Maebashi, Japan); glial fibrillary acidic protein (polyclonal; DAKO, Glostrup, Denmark); HLA-DR (monoclonal, CD68; DAKO); phosphorylated neurofilament (monoclonal SMI31; Sternberger Immunochemical, Baltimore, Md); myelin basic proteins (polyclonal, DAKO); and ubiquitin (polyclonal, DAKO) antibodies.

In addition to the routine neuropathologic examination mentioned above, studies were performed at sites of MR imaging signalintensity abnormalities or atrophy to correlate the radiologic and pathologic findings. To compare them with MR imaging findings, 2 neuropathologists investigated the following points in addition to routine examinations: 1) the macroscopic presence or absence, laterality, and localization of cerebral atrophy at the time of sectioning the brain and after fixation, 2) the macroscopic presence or absence of atrophy of the midbrain tegmentum at the time of sectioning the brain and after fixation, 3) the presence or absence and degree of degeneration of the subthalamic nuclei, and 4) the presence or absence of a lesion in the SCWM and whether the lesion constituted primary or secondary degeneration in CBD. For brain samples, 7-mm coronal sections of the lateral region passing the mamillary body vertical to the hippocampal structure were prepared. Abnormal intensi- 

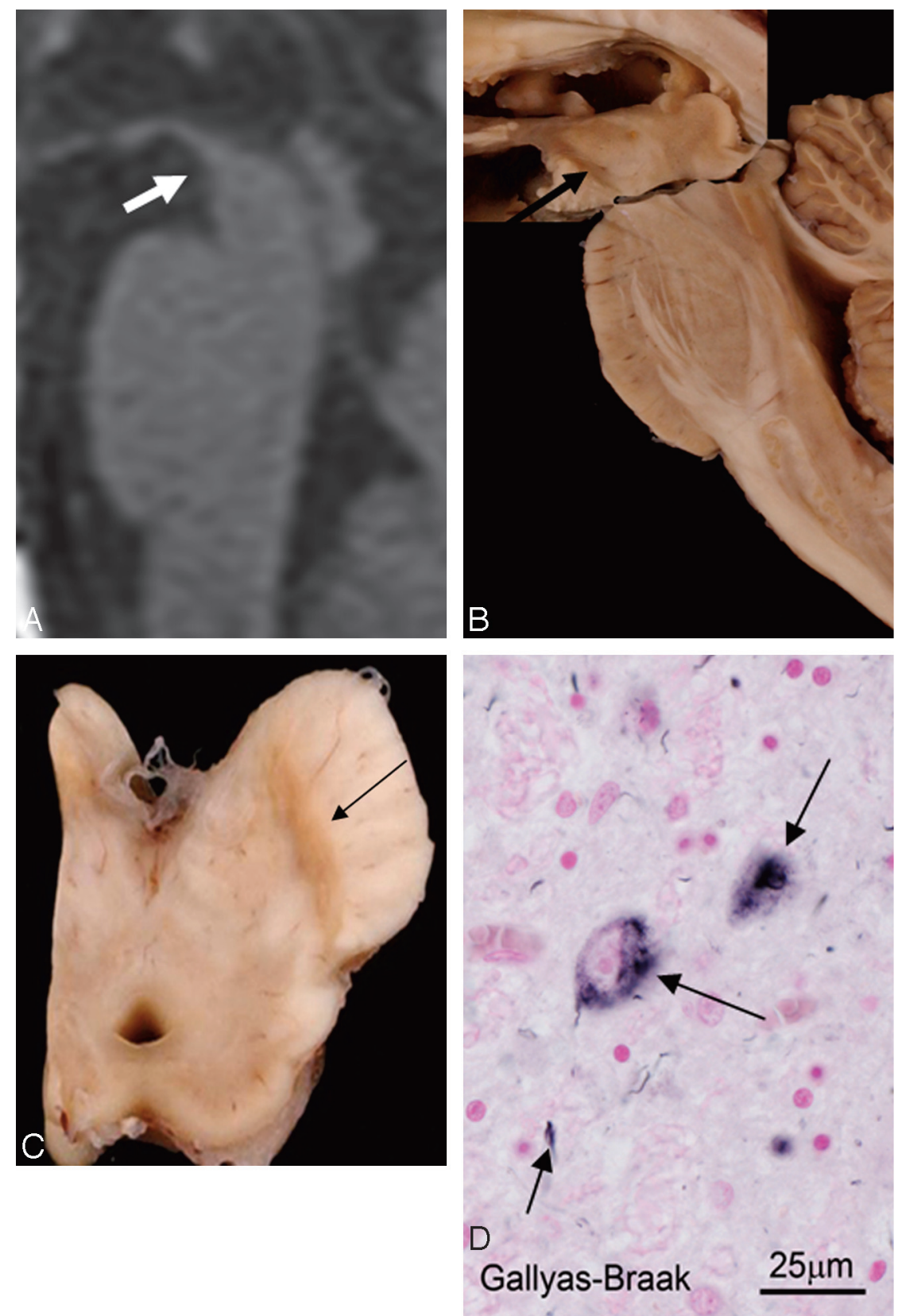

Fig 4. Corticobasal degeneration (CBD), case 1. An 84-year-old woman. A, T1-weighted midsagittal image clearly shows atrophy of the midbrain tegmentum (arrow). The area of the midbrain tegmentum is $73 \mathrm{~mm}^{2}$. B, A macroscopic specimen of the midbrain shows marked atrophy (arrow). C, A macroscopic view of the midbrain shows discoloration of the substantia nigra (arrow). $D, A$ microscopic view of the substantia nigra (Gallyas-Braak stain) shows argyrophilic threads and granular or fibrous inclusion bodies (arrows). These are consistent with CBD.

ties on MR imaging were collated with the pathologic preparations as accurately as possible, with the line passing the mamillary body vertical to the hippocampal structure as the baseline, and new pathologic sections were cut out as needed.

\section{Results}

\section{Asymmetric Cerebral Atrophy}

Asymmetric cerebral atrophy was observed in 3 of 4 patients with predominance contralateral to the more clinically affected side. In all 3 patients, cerebral atrophy affected the area including the central sulcus. In 1 patient, the radiologic and pathologic findings of predominantly frontal lobe atrophy were correlated (case 1, Fig $1 A,-B$ ). In 1 patient (case 2, Fig $2 A$,
$-B$ ) in whom the interval between MR imaging and autopsy was 3 years, asymmetric cortical atrophy was difficult to detect on MR imaging, but frontal atrophy with some asymmetry was seen on autopsy. In this patient, clinical evaluation revealed no asymmetric cortical symptoms, so the clinical diagnosis was Alzheimer disease. In the 13 cases with PSP, excluding 1 patient, no asymmetric atrophy was noted.

\section{Atrophy of Midbrain Tegmentum}

On midsagittal sections, by using the method of Oba et al, ${ }^{2}$ the mean area of the midbrain tegmentum of the 4 patients with CBD was $67.6 \pm 7.4 \mathrm{~mm}^{2}$ (range, $62.3-73.3 \mathrm{~mm}^{2}$ ) (Fig 3; case 1 , Fig $4 A-D$ ), which is markedly less than normal, with the 


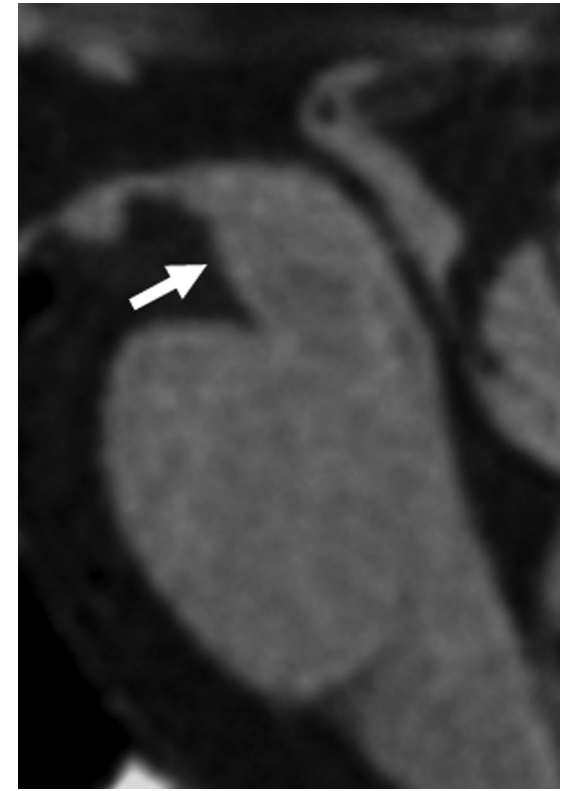

Fig 5. An age-matched healthy control 72-year-old woman. T1-weighted midsagittal image shows no obvious atrophy of the midbrain tegmentum (arrow). The area of the midbrain tegmentum is $128 \mathrm{~mm}^{2}$.

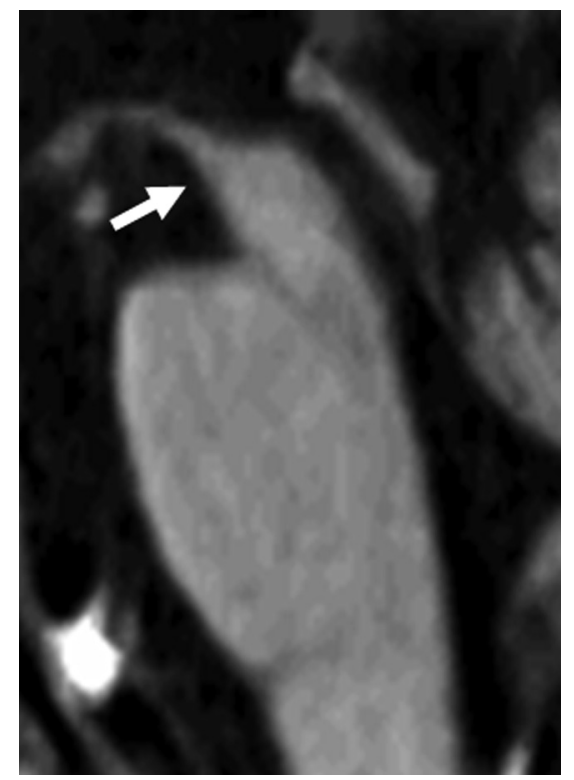

Fig 6. Progressive supranuclear palsy. A 74-year-old man. T1-weighted midsagittal image clearly shows atrophy of the midbrain tegmentum (arrow). The area of the midbrain tegmentum is $71 \mathrm{~mm}^{2}$.

mean area of $123.8 \pm 10.8 \mathrm{~mm}^{2}$ in the controls (range, $108.0-$ $132.4 \mathrm{~mm}^{2}$ ) (Figs 3 and 5). In the 13 patients with neuropathologically confirmed PSP, the mean area was $70.7 \pm 12.1 \mathrm{~mm}^{2}$ (range, 58.6-89.8 $\mathrm{mm}^{2}$ ) (Figs 3 and 6). Although statistical analysis was not possible because of the small number of cases, there was no individual overlap of the midbrain tegmental area between the healthy controls and patients with CBD and PSP, apparently showing that severe atrophy of the midbrain tegmentum was present in CBD and PSP. On neuropathologic examination, there was also atrophy of the midbrain tegmentum and marked depigmentation of the substantia nigra and locus ceruleus. Other findings included melanophagia and gli- osis, and Gallyas-Braak silver staining revealed argyrophilic threads and granular or fibrous inclusion bodies. These findings were consistent with CBD. In the pontine tegmentum and oculomotor and trochlear nuclei, many AT8-immunoreactive pretangles were observed.

\section{SCWM Signa-Intensity Change on T2-Weighted Images and FLAIR}

In all 4 patients with CBD, T2-weighted images and FLAIR showed diffuse high-intensity signals in the SCWM. In 3 patients, high signal intensity in the SCWM was recognized on the predominantly atrophic side (case 1 , Fig $7 A-C$ ). In 1 patient, a high signal intensity was noted bilaterally over a wide area in the frontal lobes (case 3, Fig. $8 A-C$ ). Corresponding to the sites of white matter lesions, myelin sheath staining was decreased, and these sites were stained positively for antiphosphorylated tau antibody. These changes are primary characteristic of CBD. On neuropathologic examination, there was some involvement of U-fibers, but because of image-quality limitations on MR imaging, U-fiber involvement could not be specifically detected. In the 13 patients with PSP, there was no diffuse signal-intensity abnormality in the SCWM. There was no neuropathologic finding indicating primary degeneration of the SCWM.

\section{Symmetric High Signal Intensity Bilaterally in Subthalamic Nuclei on T1-Weighted Images}

T1-weighted MR images showed symmetric high signal intensity bilaterally in the subthalamic nuclei in all 3 patients in whom T1-weighted images were obtained (case 1, Fig 9A-C). In the remaining patient, the signal intensity in the subthalamic nuclei was not evaluated because no T1-weighted coronal spin-echo images could be obtained. Spoiled gradient echo (TR/TE, 21/6 ms; TI, $0 \mathrm{~ms}$; flip angle, $20^{\circ}$ ) was selected instead. These sites showed a brownish change on macroscopic examination, and on microscopic examination, antiphosphorylated tau antibody-positive neurons and gliosis were observed. These changes were characteristic of CBD. MR imaging signal-intensity changes were also present in 4 of 7 patients with PSP in whom T1-weighted images were obtained (Fig $10 A-C$ ). Because neuropathologically examined cases were retrospectively evaluated, signal-intensity evaluation was limited to visual examination by 2 neuroradiologists. It was difficult to distinguish CBD and PSP on imaging, and no asymmetry was identified. However, on neuropathologic examination, degeneration of the subthalamic nuclei was more severe in PSP than in CBD.

\section{Discussion}

In 3 of the 4 patients with CBD, atrophy was predominantly contralateral and extended to the central sulcus. On neuropathologic examination, the MR imaging findings of atrophy were confirmed. In 1 patient with no asymmetric cortical symptoms, there was no asymmetry on MR imaging (case 2, Fig $3 A,-B$ ) and the clinical diagnosis was Alzheimer disease. In this patient, in whom the interval between the last MR imaging and autopsy was 3 years, neuropathologic examination did show asymmetric atrophy, but it was mild compared with that in the other 3 patients. In some patients, CBD presents with dementia, behavioral abnormalities, and attention deficit in 

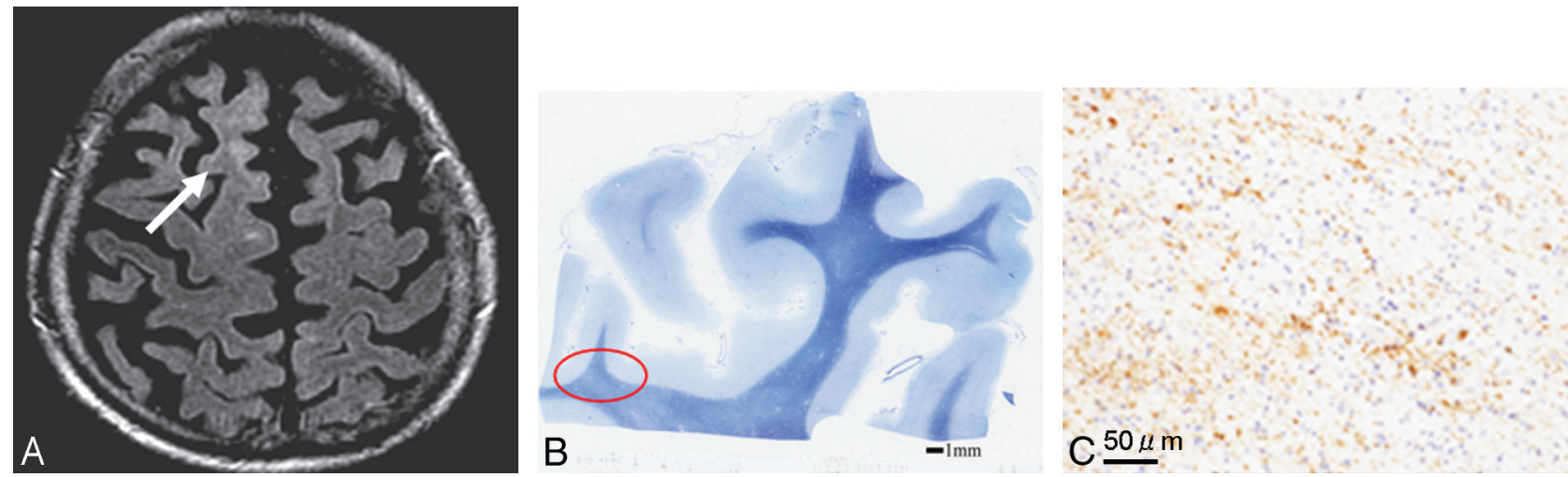

Fig 7. Corticobasal degeneration (CBD), case 1. An 84-year-old woman. A, Axial T2-weighted image shows a high signal intensity in the right frontal subcortical white matter (white arrow). $B$, In a microscopic specimen of the right frontal lobe corresponding to the site of white matter lesions, myelin sheath staining is decreased (red oval). The scale is $1 \mathrm{~mm}$. $C$, In this area, there is positive staining for antiphosphorylated tau antibody on AT8 staining, which is compatible with the primary changes in CBD. The scale is $50 \mu \mathrm{m}$.
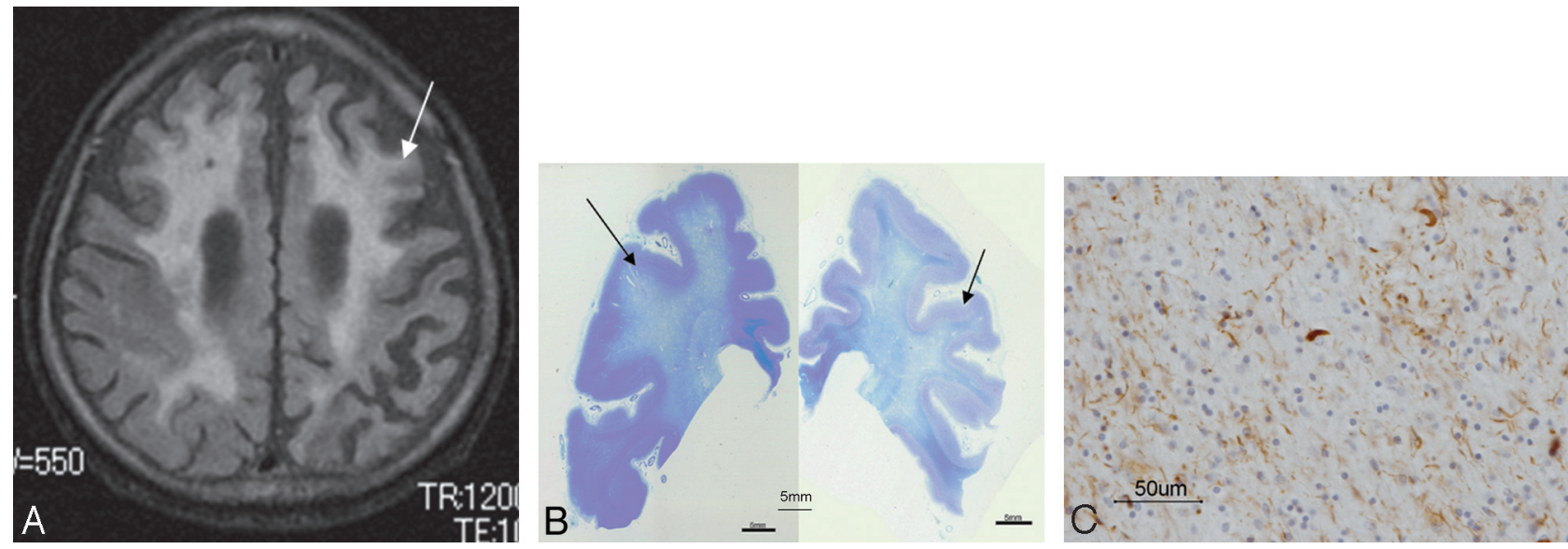

Fig 8. Corticobasal degeneration (CBD), case 3. A 70-year-old man. A, An axial fluid-attenuated inversion recovery image shows a high signal intensity bilaterally over a wide area in the frontal lobes (arrow). B, Corresponding to sites of white matter lesions, myelin sheath staining is decreased (arrow). The scale is $5 \mathrm{~mm}$. C, These sites are stained positively for antiphosphorylated tau antibody. The scale is $50 \mu \mathrm{m}$. The changes are primary characteristics of CBD.

the early stage, but patients may be misdiagnosed due to a lack of cortical symptoms. ${ }^{10-14}$ The above patient (case 2) may be an example of such a clinical presentation. Clinical evaluation showed little evidence of asymmetric cortical dysfunction, and neuropathology revealed only minimal cortical asymmetry. In this case, MR imaging showed a slight high signal intensity in the frontal SCWM; the midbrain tegmentum was severely atrophied, with an area of $71 \mathrm{~mm}^{2}$; and T1-weighted imaging showed symmetric high-intensity signals in the subthalamic nucleus. Although a clear description is difficult on the basis of only 1 case, it was demonstrated by the pathologic findings that when identification of the cortical sign is difficult and unilateral atrophy is unclear on imaging, imaging findings, such as atrophy of the midbrain tegmentum, an abnormal signal intensity in the SCWM on FLAIR, or signal intensity changes in the subthalamic nuclei on T1-weighted imaging, may serve as supportive findings suggesting CBD.

CBD is important to differentiate from PSP. Oba et $\mathrm{al}^{2}$ described a convenient and objective approach to diagnosing PSP based on midsagittal measurement of the midbrain tegmentum area. However, Koyama et $\mathrm{al}^{1}$ also reported midbrain tegmentum atrophy in CBD. In our 4 cases of neuropathologically confirmed disease, severe midbrain tegmentum atrophy was also observed. Although there was a limited number of cases, because there was no individual overlap of the midbrain tegmental area between the healthy controls and patients with pathologically confirmed CBD and PSP, investigation of midbrain tegmental atrophy may have led to the diagnosis of CBD and PSP. Although no statistical analysis was performed because of the limited number of cases, it may be difficult to differentiate CBD from PSP on the basis of the presence of midbrain tegmental atrophy alone. A so-called "penguin sign" may be a distinguishing feature of PSP, but with severe midbrain tegmentum atrophy, both PSP and CBD must be considered. On neuropathology, there was marked depigmentation of the substantia nigra and locus ceruleus. Other findings included melanophagia and gliosis, and Gallyas-Braak silver staining revealed argyrophilic threads and granular or fibrous inclusion bodies. These findings were consistent with CBD as a cause of the atrophy. The degeneration of motor nerve nuclei, including the oculomotor and abducens nuclei, in the brain stem tegmentum was also noted, but no corresponding imaging findings were seen in the present study.

The localization of SCWM abnormalities, though different from those in previous reports, ${ }^{1,15}$ was prominent in the present study. In CBD, Tokumaru et al, ${ }^{15}$ Doi et al, ${ }^{16}$ and Koyama et $\mathrm{al}^{1}$ described predominantly unilateral SCWM abnormalities on T2-weighted imaging and FLAIR. In their re- 

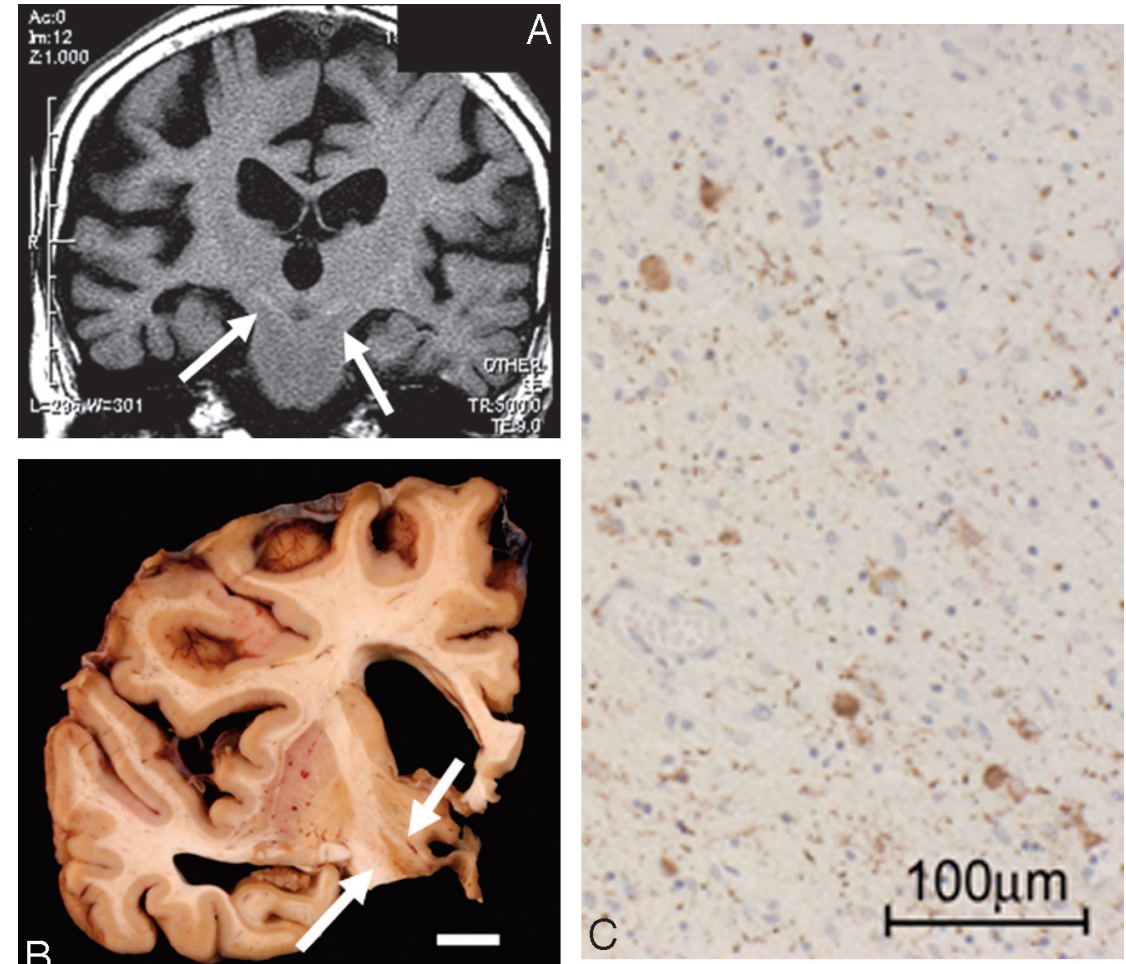

Fig 9. Corticobasal degeneration (CBD), case 1. An 84-year-old woman. A, Coronal T1-weighted image shows symmetric high signal intensity bilaterally in the subthalamic nuclei (arrows).B, A macroscopic specimen shows a brownish change in the subthalamic nuclei (arrows). C, On microscopic examination (AT8 stain), antiphosphorylated tau antibody-positive neurons and gliosis are observed. These changes are characteristic of CBD. The scale is $100 \mu \mathrm{m}$.
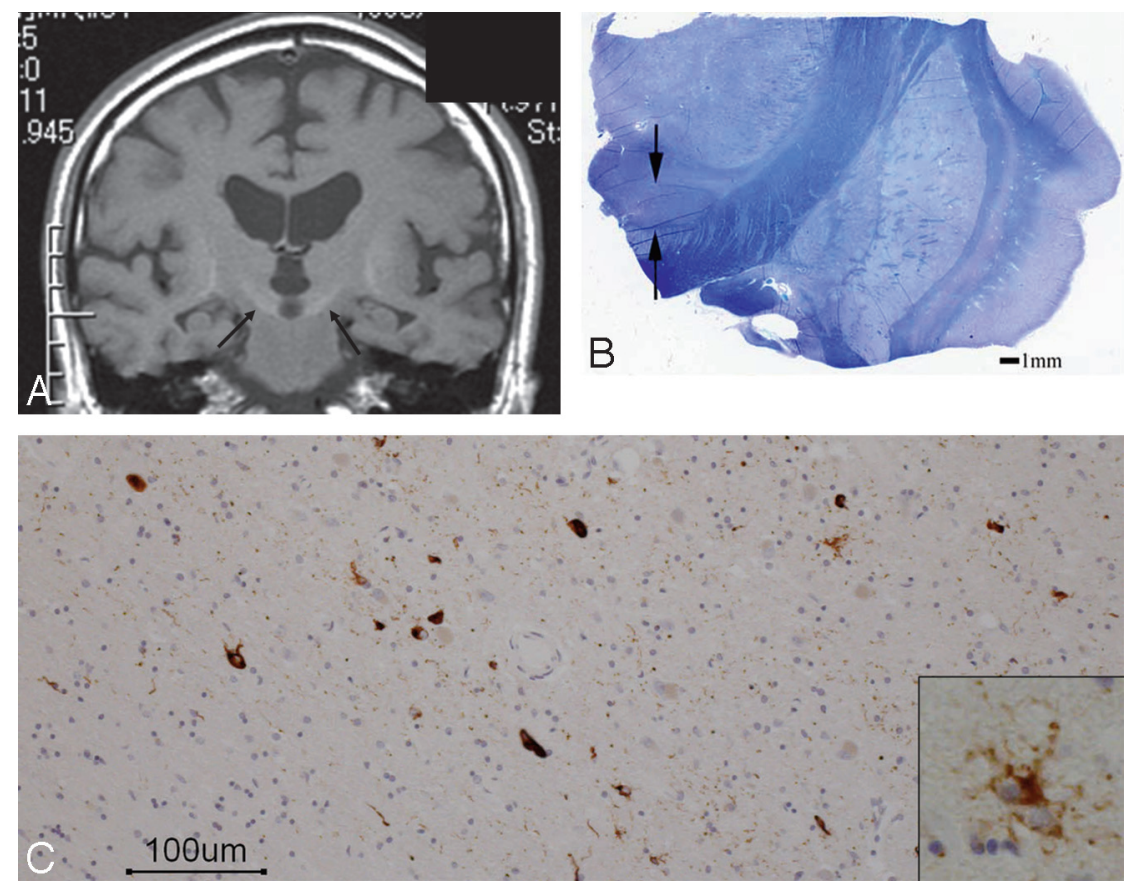

Fig 10. Progressive supranuclear palsy (PSP). An 84-year-old man. $A$, Coronal T1-weighted image shows a symmetric high signal intensity bilaterally in the subthalamic nuclei (arrow). $B$, A microscopic specimen of myelin-sheath staining shows the atrophic change of the subthalamic nuclei (arrows). The scale is $1 \mathrm{~mm}$. $C$, On microscopic examination in the subthalamic nuclei, AT8 staining is clearly positive in the neurons (brown area). An enlarged image shows a tuft. These changes are characteristic of PSP. The scale is $100 \mu \mathrm{m}$.

ports, localization in the precentral gyrus of the SCWM and signal-intensity abnormalities contralateral to the clinically affected side were noted. ${ }^{1,15}$ However, in the present study, the localization of white matter signal-intensity abnormalities differed among the 3 cases. In particular, in case 3, T2-weighted imaging and FLAIR showed widespread signal-intensity changes in the frontal lobe white matter bilaterally. This appearance differed from that reported by Doi et $\mathrm{al}^{16}$ and Koyama et al..$^{1}$ In 2 patients, signal-intensity abnormalities were present in the superior frontal gyrus white matter. Only 1 
patient showed signal-intensity abnormalities in the precentral gyrus SCWM. It is important to recognize the possibility of CBD showing different localizations of atrophy and white matter signal-intensity abnormalities rather than findings in the more typical cases as reported by Koyama et al. ${ }^{1}$ In cases without predominantly unilateral cortical signs on clinical evaluation, the localization of atrophy and SCWM signal-intensity abnormalities may differ from typical cases. In these cases, MR imaging findings of high signal intensity in the subthalamic nucleus on T1-weighted images and severe atrophy of the midbrain tegmentum on midsagittal sections can provide useful information.

Rebeiz et $\mathrm{al}^{17}$ first reported CBD with distinct features and a clinically asymmetric onset characterized by apraxia, dystonia, postural instability, and an akinetic-rigid syndrome that does not respond to levodopa, but since then, cases presenting with dementia, in which Alzheimer or Pick disease must be ruled out, and cases with other clinical features, in which PSP must be ruled out, have been reported. ${ }^{18-21}$ A variety of underlying neuropathologic features have also been reported. Recent reports clarified the presence of many phenotypes of CBD neuropathologically and clinically. ${ }^{10-14}$ Although there were only 4 cases, it was difficult to diagnose CBD clinically in cases definitely diagnosed neuropathologically, and 1 case showed imaging findings different from those previously reported. The lesions in the white matter widely expanded bilaterally in case 3 , indicating that the clinical features alone did not confirm CBD. This case probably represents a new clinical phenotype of CBD.

Neuropathologic examination of SCWM lesions that correlated with the MR imaging findings showed the typical tauopathy of CBD. The degenerative changes were characteristic of CBD. In 1996, we suggested, on the basis of the neuropathologic findings in $\mathrm{CBD}$, that white matter lesions of the frontal lobe were secondary degenerative changes. ${ }^{15}$ Doi et $\mathrm{al}^{16}$ and Koyama et $\mathrm{al}^{1}$ also believe that these white matter lesions in CBD reflect the progression of neuronal degeneration, especially demyelination secondary to axonal loss or change. However, in the present study, at sites where MR imaging showed white matter lesions, though neuropathologic examination revealed some secondary degeneration, tauopathy in the white matter, particularly the SCWM, was clearly evident. This shows a positive radio-pathologic correlation of these changes in CBD. In 2002, Dickson et $\mathrm{al}^{8}$ proposed neuropathologic criteria, with a primary emphasis on tau-positive neuronal and glial lesions, for the diagnosis of CBD. Advances in neuropathologic staining methods to evaluate tau have expanded our knowledge of these primary changes in CBD. Dickson et al also confirmed tauopathy of the white matter in CBD. After the report of Dickson et al, the neuropathologic evaluation criteria for lesions in the white matter apparently changed, and the finding that lesions in the white matter were primary degeneration in $\mathrm{CBD}$, not secondary, may be important new information for the evaluation of an association with clinical and pathologic findings. In the present cases, degeneration was severe, affecting U-fibers, which should be investigated to collate with MR images.

T1-weighted images, obtained in 3 patients with CBD, showed symmetric high-intensity signals in the subthalamic nucleus. This finding was also present in a high proportion of patients with PSP, making an MR imaging-based differential diagnosis difficult. Neuropathology of sites corresponding to these signal-intensity changes showed tauopathy-related degeneration. Gliosis was also present, but on T2-weighted imaging and FLAIR, signal-intensity changes were difficult to detect. Only axial sections were obtained on T2-weighted imaging. These may have been insufficient to delineate the subthalamic nucleus adequately. In PSP, there was a high rate of similar findings, so these were not useful in the differential diagnosis. In all patients with PSP, the neuropathology showed more severe degeneration than in those with CBD. In addition to midbrain tegmentum atrophy, the localization of degeneration was similar in CBD to that seen in PSP. These signal-intensity changes were only visually evaluated by the neuroradiologists in the limited number of cases, but no signal-intensity change was noted in the pathologically healthy group, suggesting that primary degenerative findings of the individual diseases in the subthalamic nuclei and abnormal signals in this region are significant to some extent. However, differentiating CBD and PSP based on these findings alone is not possible. Therefore, a combination of imaging findings, including the presence of white matter signal-intensity changes and asymmetric atrophy, is important in correctly diagnosing CBD.

\section{Conclusions}

The correlation between radiologic and pathologic findings was investigated in patients with $\mathrm{CBD}$, and MR imaging findings that could be used to differentiate CBD from PSP clinically were identified.

In $\mathrm{CBD}$, midbrain tegmentum atrophy was severe, and degeneration in this area was correlated with the severity. However, this finding did not help in the differential diagnosis distinguishing CBD from PSP. T1-weighted imaging showed symmetric high-intensity signals in the subthalamic nucleus, but a large proportion of patients with PSP had a similar finding. On neuropathologic examination, each disorder showed characteristic degeneration. The degree of degeneration was more severe in PSP, but no imaging-based differences were observed. In CBD, there was a high rate of atrophy contralateral to the clinically affected side, with extension to the central sulcus. This suggests that the localization of atrophy differs depending on the underlying etiology. In PSP, unilateral atrophy was not a predominant finding.

MR imaging, FLAIR, and T2-weighted imaging showed high signal intensities in the SCWM. Previous studies have correlated this finding with secondary degeneration. The present study is the first to correlate these SCWM signal-intensity changes with primary degeneration in CBD. In addition, the localization of white matter lesions was correlated with a variety of clinical phenotypes. This suggests that there are $\mathrm{CBD}$ types other than those that are localized only to the precentral gyrus SCWM.

\section{References}

1. Koyama M, Yagishita A, Nakata Y, et al. Imaging of corticobasal degeneration syndrome. Neuroradiology 2007;49:905-12

2. Oba $H$, Yagishita A, Terada $H$, et al. New and reliable MRI diagnosis for progressive supranuclear palsy. Neurology 2005;28:2050-55

3. Saito Y, Murayama S. Neuropathology of mild cognitive impairment. Neuropathology 2007;27:578-84

4. Mirra SS, Heyman A, McKeel D, et al. The Consortium to Establish a Registry 
for Alzheimer's Disease (CERAD). Part II. Standardization of the neuropathologic assessment of Alzheimer's disease. Neurology 1991;41:479-86

5. Saito Y, Ruberu NN, Sawabe M, et al. Lewy body-related alpha-synucleinopathy in aging. J Neuropathol Exp Neurol 2004;63:742-49

6. McKeith IG, Galasko D, Kosaka K, et al. Consensus guidelines for the clinical and pathologic diagnosis of dementia with Lewy bodies (DLB): report of the consortium on DLB international workshop. Neurology 1996;47:1113-24

7. Braak H, Braak E. Neuropathological stageing of Alzheimer-related changes. Acta Neuropathol 1991;82:239-59

8. Dickson DW, Bergeron C, Chin SS, et al. Office of Rare Diseases Neuropathologic Criteria for corticobasal degeneration. J Neuropathol Exp Neurol 2002;61:935-46

9. Hauw JJ, Daniel SE, Dickson D, et al. Preliminary NINDS neuropathologic criteria for Steele-Richardson-Olszewski syndrome (progressive supranuclear palsy). Neurology 1994;44:2015-19

10. Mathuranath PS, Xuereb JH, Bak T, et al. Corticobasal ganglionic degeneration and/or frontotemporal dementia? A report of two overlap cases and review of literature. J Neurol Neurosurg Psychiatry 2000;68:304-12

11. Grimes DA, Lang AE, Bergeron CB. Dementia as the most common presentation of cortical-basal ganglionic degeneration. Neurology 1999;53:1969-74

12. Schneider JA, Watts RL, Gearing M, et al. Corticobasal degeneration: neuropathologic and clinical heterogeneity. Neurology 1997;48:959:969
13. Rinne JO, Lee MS, Thompson PD, et al. Corticobasal degeneration: a clinical study of 36 cases. Brain 1994;117:1183-96

14. Bergeron C, Pollanen MS, Weyer L, et al. Unusual clinical presentations of cortical-basal ganglionic degeneration. Ann Neurol 1996;40:893-900

15. Tokumaru AM, O'uchi T, Kuru Y, et al. Corticobasal degeneration: MR with histopathologic comparison. AJNR Am J Neuroradiol 1996;17:1849-52

16. Doi T, Iwasa K, Makifuchi T, et al. White matter hyperintensities on MRI in a patient with corticobasal degeneration. Acta Neurol Scand 1999;99:199-201

17. Rebeiz JJ, Kolodny EH, Richardson EP Jr. Corticodentatonigral degeneration with neuronal achromasia: a progressive disorder of late adult life. Trans Am Neurol Assoc 1967;92:23-26

18. Brown J, Lantos PL, Roques P, et al. Familial dementia with swollen achromatic neuron and corticobasal inclusion bodies: a clinical and pathological study. J Neurol Sci 1996;135:21-30

19. Doran M, du Plessis DG, Enevoldson TP, et al. Pathological heterogeneity of clinically diagnosed corticobasal degeneration. J Neurol Sci 2003;216:127-34

20. Feany MB, Mattiace LA, Dickson DW. Neuropathologic overlap of progressive supranuclear palsy, Pick's disease, and corticobasal degeneration. J Neuropathol Exp Neurol 1996;55:53-67

21. Jendroska K, Rossor MN, Mathias CJ, et al. Morphological overlap between corticobasal degeneration and Pick's disease: a clinicopathological report. Mov Disord 1995;10:111-14 